\title{
Successful aging: Is there hope?
}

\author{
Bradley Willcox MD MSc
}

See related research article by Sabia and colleagues on page 1985 and at www.cmaj.ca/lookup/doi/10.1503/cmaj.121080

$\mathrm{T}$ he world's population is aging rapidly, the consequences of which will be substantial. By 2031, more than $20 \%$ of Canadians will be senior citizens. ${ }^{1,2}$ This trend risks overwhelming health and social support systems with millions of older adults, many of whom will have chronic pain, serious agerelated diseases and disability. ${ }^{3}$

What advice can we give our patients to optimize their odds of aging with better health? Is there reason for hope? This is a challenging issue, with few evidence-based studies attempting to quantify healthy aging. ${ }^{4}$ Such studies must follow thousands of participants - usually for several decades - and must measure a variety of risk factors and aging-related outcomes to achieve reasonable statistical power and address the complexity of aging. The article by Sabia and colleagues features some fascinating findings from one such study - the UK-based Whitehall II Study. ${ }^{5}$

The Whitehall II Study began recruiting participants in 1985-1988 for an observational epidemiologic study. ${ }^{5}$ The study has clinically examined (at intervals of about $5 \mathrm{yr}$ ) a cohort of 10308 British men and women, aged 35-55 years at baseline, for various health outcomes, including diseases and disabilities that typically occur with aging. Sabia and colleagues ${ }^{5}$ used these data and focused on assessing successful aging for 5100 healthy, middle-aged (range 42$63 \mathrm{yr}$ ), men and women assessed during the 1991-1994 examination cycle. Although healthy aging was the primary outcome, the authors also studied survival. Because there is no consensus definition for successful aging, which is one of the reasons it has been challenging to study, the authors adapted the classical definition from Rowe and Kahn's work. ${ }^{6}$ Successful aging was defined as being alive at 60 years of age and beyond; having no history of coronary artery disease, cancer, stroke or diabetes; good cognitive, physical, respiratory and cardiovascular function without disability; and good mental health. Sabia and colleagues subsequently assessed whether engaging in 4 healthy behaviours during midlife influences a person's ability to age successfully.
Their results were quite remarkable and do indeed provide some reason for hope. Building on earlier work by Willcox and colleagues, ${ }^{7}$ who found that 9 factors had additive effects on odds of successful aging over a follow-up of 4 decades, Sabia and colleagues ${ }^{5}$ attempted to further quantify odds of successful aging based on 4 healthy behaviours: regular physical activity, consuming fruits and vegetables daily, drinking alcohol moderately and never smoking. Over a 16-year follow-up, the odds for successful aging increased for participants engaging in any individual healthy behaviour. ${ }^{5}$ Collectively, up to $47 \%$ of the population attributable risk (the portion of successful aging explained by exposure to the particular risk factors) was explained by these 4 healthy behaviours. ${ }^{5}$ Furthermore, the beneficial impact of healthy behaviours increased linearly, with $33 \%$ increased odds for successful aging for each additional healthy behaviour. ${ }^{5}$ Overall results did not vary markedly between men and women.

Moderate alcohol consumption and not smoking have well-known health benefits, so it is not surprising that they may affect odds for successful aging. ${ }^{47,8}$ However, the apparent benefits of physical activity and healthy diets, which were not always evident in previous studies, were impressive. There was a relatively large beneficial effect from physical activity $(\geq 2.5 \mathrm{~h} / \mathrm{wk}$ of moderate activity, or $\geq 1 \mathrm{~h} / \mathrm{wk}$ of vigorous activity), which supports the notion that even small amounts of regular activity may have important health effects. Similarly, although the number of

\section{- Ker POINTS}

- Sabia and colleagues found that 4 behaviours increase the odds of living in good health beyond 60 years of age: regular physical activity, eating fruits and vegetables daily, drinking alcohol moderately and never smoking.

- Together, these behaviours accounted for almost half the potential for successful aging.

- Several ongoing cohort studies of aging are likely to yield additional insights in the future.

- Genomic, epigenomic, proteomic and metabolomic studies are just beginning to assess the determinants of healthy aging. 
fruits and vegetables to be eaten daily was not quantified, consumption of at least some amount each day appears to have an important association with successful aging. It would be interesting to know whether more servings of fruits and vegetables has a stronger effect and whether there is a dose-response relation.

Strengths of this study include the large number of participants $(n=5100)$, a relatively long follow-up (median $16.3 \mathrm{yr}$ ), detailed and strong analyses and the focus on successful aging as the primary outcome. Most studies of healthy aging have focused on less comprehensive outcomes, such as a particular disease or disability. ${ }^{4} \mathrm{Al}-$ though such studies are important, the collective outcome (maintaining relatively good health and high functional ability) may be most important to the aging patient and, ultimately, is an important determinant of the impact of aging on health care and social systems. ${ }^{3}$

Despite the limitations often seen in large, observational cohort studies, research such as this is valuable for assessing the complex nature of human aging. Interventional studies of aging that can show causation are possible in model organisms with limited lifespans (e.g., yeast, worms and mice), but are impractical to perform with humans. Of note, that only $12 \%$ of the participants engaged in all 4 healthy behaviours suggests we may have much to gain by continuing to encourage our patients in such habits. More work on the social or other determinants as to why so few people engaged in all 4 behaviours may be required.

The future for research on human aging is bright and should continue to yield results that can influence clinical decision-making and health policy. Several epidemiologic cohort studies have been established around the world to prospectively assess human aging, and they are continuing to enrich our knowledge base. ${ }^{8}$ Other studies, such as the Cardiovascular Health Study and the Honolulu Heart Study, are among several large cohort studies that were originally established to investigate other outcomes, but which now have major ancillary studies of aging. ${ }^{8}$ In Canada, in addition to several longstanding studies of aging, ${ }^{8}$ an effort to recruit 50000 participants is underway for a novel prospective study of the environmental, genetic and epigenetic determinants of human aging, the Canadian Longitudinal Study on Aging. ${ }^{9}$ Still other initiatives are re-examining the concept of successful aging itself and establishing a more comprehensive, interdisciplinary approach that is fostering novel research. ${ }^{10,11}$

Finally, genomic, epigenomic, proteomic and metabolomic studies are just beginning to assess the genetic and environmental components of healthy aging with systems approaches, which will ultimately help us better understand the complex relations that determine the odds of healthy aging. Such a plethora of novel initiatives and diverse approaches should engender hope that we are on the verge of discoveries that will lead to a much healthier old age. But why wait? Engaging in healthy behaviours now could give us a head start.

\section{References}

1. Estimates of population, by age group and sex for July 1 , Canada, provinces and territories, annual [table 051-0001]. Ottawa (ON): Statistics Canada, 2011

2. Projected population, by projection scenario, sex and age group as of July 1, Canada, provinces and territories, annual [table 052-0005]. Ottawa (ON): Statistics Canada, 2011.

3. Sinha SK. Why the elderly could bankrupt Canada and how demographic imperatives will force the redesign of acute care service delivery. Healthc Pap 2011;11:46-51.

4. Depp CA, Jeste DV. Definitions and predictors of successful aging: a comprehensive review of larger quantitative studies. Am J Geriatr Psychiatry 2006;14:6-20.

5. Sabia S, Singh-Manoux A, Hagger-Johnson G, et al. Influence of individual and combined healthy behaviours on successful aging. CMAJ 2012;184:1985-92.

6. Rowe JW, Kahn R. Human aging: usual and successful. Science 1987;237:143-9.

7. Willcox BJ, He Q, Chen R, et al. Midlife risk factors and healthy survival in men. JAMA 2006;296:2343-50.

8. Stanziano DC, Whitehurst M, Graham P, et al. A review of selected longitudinal studies on aging: past findings and future directions. J Am Geriatr Soc 2010;58(Suppl 2):S292-7.

9. Canadian Longitudinal Study on Aging (CLSA). Hamilton (ON): The Study; 2009. Available: www.clsa-elcv.ca (accessed 2012 Oct. 21).

10. Poon L, Fry C. Kahana E, et al. Healthy successful aging: a public health mandate. Athens (GA): University of Georgia. Available: www.publichealth.uga.edu/geron/research/healthy-successful-aging-public-health-mandate (accessed 2012 Oct. 23).

11. MacArthur Foundation Studies. Available: www.macfound.org /networks/research-network-on-an-aging-society (accessed 2012 Oct. 21).

Affiliation: Bradley Willcox is with the Department of Geriatric Medicine, John A. Burns School of Medicine, University of Hawaii; and the Kuakini Medical Center, Honolulu, Hawaii. 